\title{
Narrow Band HI System for the Parkes Telescope Multibeam Package
}

\author{
Raymond Haynes, Lister Staveley-Smith \\ Australia Telescope National Facility, CSIRO, PO Box 76, Epping 1710, \\ NSW, Australia \\ Ulrich Mebold, Peter Kalberla \\ RAIUB, University of Bonn, Bonn, Germany
}

Keith Jones

Physics Department, University of Queensland, Australia

Graeme White, Paul Jones, Miroslav Filipovic

University of Western Sydney, Nepean, Australia

John Dickey

Astronomy Dept., Univ. of Minnesota, Minneapolis, MN 55455, USA

Anne Green

School of Physics, University of Sydney, Sydney, Australia

\begin{abstract}
A narrow-band translator and filter system has been constructed for the Parkes Multibeam receiver. This brief note outlines the characteristics of the system and indicates the range of new projects that will be possible in studies of the Magellanic Clouds.
\end{abstract}

\section{Introduction}

In a proposal funded by the ATNF, the University of Queensland and the University of Bonn, the ATNF has now developed a narrow-band back-end system for the Parkes Multi-Beam system.

The project has provided a narrow-band back-end filter and correlationsystem able to use the inner 7-beams of the multi-beam system for observations of spectral lines over 2048 channels with a total bandwidth across each spectrum of either 4 or $8 \mathrm{MHz}$. This will provide a spectral resolution of down to $0.4 \mathrm{~km}$ $\mathrm{s}^{-1}$ for each of the two polarization channels on the inner 7-beams of the multibeam system.

This makes an important addition to the multi-beam system which can then be used to obtain "zero-spacing" data at Parkes on the multi-beam system 
to complement Australia Telescope Compact Array (ATCA) data or to map larger areas of the sky in $\mathrm{HI}$ with the observing speed-advantage obtained by multi-beaming the Parkes Telescope.

\section{System Characteristics}

The new Parkes narrow-band HI system will provide:

1. 7 beams on the sky;

2. 14.4 beam-width on each beam;

3. $\mathrm{T}_{s}$ on the sky on each beam $\sim 24 \mathrm{~K}$

4. 2 orthogonal polarizations per beam;

5. 14 IF channels of 4 or $8 \mathrm{MHz}$ bandwidth;

6. 2048 channels of spectral resolution;

7. velocity coverage is -800 to $800 \mathrm{~km} \mathrm{~s}^{-1}$ (in $8 \mathrm{MHz}$ bandwidth);

8. velocity resolution down to $0.4 \mathrm{~km} \mathrm{~s}^{-1}$ per channel.

\section{Projects}

Initial projects being undertaken with the facility by the team will include: a study of the stray-radiation problems at $21-\mathrm{cm}$ wavelength with the Parkes Telescope and the multi-beam system preparatory to using the facility for mapping of $\mathrm{HI}$ in the Galaxy; a project to map the $\mathrm{HI}$ in a test region along the Galactic Plane; and a project to survey the extended HI in the LMC region at Parkes.

In the future other feasible projects with the system will include: fully sampled surveys of the Magellanic System (including the the LMC and SMC, Magellanic Bridge and Stream); gas flow into the Magellanic Stream from the Magellanic Bridge; searches for HI gas in the neighborhood of the discrete HI clouds in the Magellanic Stream; projects related to the interaction of Magellanic Cloud gas and HI in the halo of the Galaxy ${ }^{1}$; high-velocity HI cloud ${ }^{1}$ studies; surveys of $\mathrm{HI}$ in the Galactic plane ${ }^{1}$; rotation curves of larger galaxies discovered in the HIPASS and ZOA surveys; simultaneous HI and continuum polarization studies at $1420 \mathrm{MHz}$; HI observations of newly discovered galaxies in the Zone of Avoidance survey ${ }^{1}$; HI observations of galaxies found in the HIPASS multibeam survey; and "zero-spacing" data acquisition to complement HI studies on the ATCA.

Acknowledgments. We would like to thank the ATNF's electronics, receiver and computing staff for their support in developing the narrow-band system for the Parkes multi-beam system.

\footnotetext{
${ }^{1}$ this project requires a good understanding of stray-radiation effects at the Parkes Telescope
} 